\title{
Arbor
}

\section{El Hispanismo que viene: Estados Unidos y Canadá}

\author{
David T. Gies
}

Arbor CLXVIII, 664 (Abril 2001), 493-511 pp.

El Hispanismo en los Estados Unidos y Canadá se encuentra en un período de gran agitación e importantes cambios. En realidad, no se puede hablar de un Hispanismo en este continente, porque el «Hispanismo» son tres fenómenos distintos e interrelacionados, cada uno con sus intereses creados, sus presiones y sus problemas, que trabajan separadamente y, a la vez, cada uno en conjunto con los otros dos. Por «Hispanismo» se entiende 1) el estudio de las letras y la cultura de España, 2) el mismo estudio referido a Latinoamérica y 3 ) el nuevo fenómeno de los estudios «US-latinos» ${ }^{1}$ (o «chicanos»), cuya definición incluye literatura escrita en inglés o en español por descendientes de gente de habla hispana pero no siempre nativos. La mezcla y equilibrio de estas tres ramas del árbol hispánico en departamentos de lengua, literatura y cultura -especialmente en los Estados Unidos- crea una situación de gran dinamismo que está marcando profundamente el futuro de nuestra profesión.

El detalle más importante que hay que tomar en cuenta al analizar el hispanismo en los Estados Unidos es el simple detalle demográfico: la minoría hispana será, dentro de veinte años, la minoría más significativa en todo el país. Como escribe Amparo Morales:

La importancia y la representatividad que han alcanzado los grupos hispanos en la sociedad actual estadounidense se hace palpable no sólo en los datos cuantitativos demográficos, sino también en las inquietudes y los temores de muchos norteamericanos. (241)

La minoría hispana ya es la minoría con el índice de crecimiento más rápido, y Estados Unidos ya es la cuarta nación en el mundo en cuanto a población de hablantes de español. «Los datos indican que en 1982 había 15,8 millones de hispanos, que representaban aproximadamente el 7 por 
ciento de la población general, y que alcanzaron ya el 11,1 por ciento en 1997, con una población de 29,7 millones» (Morales 242). Al comenzar el nuevo milenio, habrá 16,6 millones de hispanohablantes en Estados Unidos, bilingües y monolingües. Y dentro de veinte años, la población hispana añadirá más individuos a la población estadounidense cada año que todos los otros grupos étnicos juntos. Estas cifras tienen implicaciones de gran importancia para el estudio de la lengua, la literatura y la cultura de las regiones de habla española en el siglo XXI.

Naturalmente, como resultado de estas cifras, la enseñanza del español está barriendo la enseñanza de las otras lenguas, tanto en las escuelas primarias como en las secundarias y en el nivel universitario. Antes, como pasaba también en las escuelas y universidades europeas, dominaba el estudio del francés y el alemán, pero este predominio es ya en este país historia pasada. Aquí, hay más estudiantes que nunca que insisten en estudiar el español, más interés en las cosas hispanas, $\mathrm{y}$, consiguientemente, más preocupación en los departamentos de francés y alemán que ven disminuir sus cifras y, por ende, su poder respecto de los departamentos de español. En las dos mil universidades estadounidenses, el castellano se estudia más que todas las otras lenguas extranjeras juntas; el 65 por ciento de los estudiantes universitarios prefiere el español. En las escuelas secundarias, pasa lo mismo (y muchos de estos estudiantes llegarán a las puertas de las universidades): hay cuatro millones de alumnos en las clases de español, pero sólo un millón en francés y medio millón en alemán. El agregado de educación de la embajada española en Washington observa que es más fácil hallar trabajo como profesor de castellano en Nueva York que en Segovia (La Vanguardia 19 octubre 1999: 60).

Estas cifras reflejan realidades universitarias también. A nivel de estudiantes de licenciatura, la Modern Language Association of America documenta que entre 1995 y 1998, se ha experimentado un aumento del 8,3 por ciento en el número de estudiantes matriculados en cursos de español, pero un descenso del 3,1 por ciento en francés y del 7,5 por ciento en alemán (hay que notar que es un descenso menos rápido que el registrado entre 1990 y 1995). Al nivel graduado, aunque ha habido un aumento en el número de doctorandos de francés y alemán entre 1985-86 y 1996-97 (los últimos años en que la Modern Language Association of America ha publicado las estadísticas; cifras revisadas en septiembre de 1999), el aumento ha sido muy inferior al incremento experimentado en el campo de la filología española ${ }^{2}$. Por ejemplo, el número de doctorandos en el campo de la filología francesa en 1985-86 fue 102, cifra que llegó a 150 en 1996-97; en alemán, se subió mucho menos, de 79 a 83 (en 1992- 


\section{El Hispanismo que viene: Estados Unidos y Canadá}

93 hubo 105 doctorandos de filología alemana, pero ese año vio aumentos en todos los campos). El español se disparó de 122 en 1985-86 a más del doble de esa cifra -249- en 1996-97.

Hemos visto recientemente un cambio en los hábitos de contratación de muchas universidades norteamericanas, algunas de las cuales contratan a profesores temporales o sin contrato fijo, sin permanencia («tenure»), pero el número de estudiantes interesados en el campo de la filología española sigue creciendo. Estos estudiantes ahora descubren que la entrada en la plantilla de profesores universitarios es más difícil hoy en día que hace años; sin embargo, creen (esperan) que los cambios demográficos que hemos explicado arriba les abrirán paso a los puestos universitarios. En muchos casos es cierto - los mejores estudiantes se colocan bien (142 doctorandos de español consiguieron puestos con posibilidad de permanencia en 1996-97, 54 sin permanencia pero a tiempo completo, y $12 \sin$ permanencia y a tiempo parcial)_.

El poder adquisitivo de la población hispana crece más rápidamente que el de otras minorías; sólo lo superan los de etnia asiática. Todo esto tiene un impacto importante en el sistema educativo, naturalmente, porque los estudiantes de esta minoría hispana insisten ya en hablar y estudiar su propia lengua y los productos intelectuales de su cultura. Así, lo que hace cincuenta años fue el Hispanismo en los Estados Unidos -el estudio casi exclusivo de la literatura española peninsular - ya no existe. Lo que caracterizó al hispanismo peninsular en este siglo fueron, naturalmente, los traumáticos acontecimientos producidos por la Guerra Civil Española, después de la cual toda una generación de críticos vino a parar a las universidades norteamericanas. Aquellos grandes profesores crearon el currículum moderno de estudios hispánicos (en realidad, estudios peninsulares), un currículum que dominó el hispanismo estadounidense y canadiense durante dos generaciones (ver Resina 112). Ese currículum se ha abierto a la cultura latinoamericana y, ahora, a ba cultura «US-latina». Esto produce algunas tensiones en los departamentos universitarios sobre lo que se va a enseñar y estudiar, algo paralelo al famoso debate entre los Antiguos y los Modernos de finales del siglo XVII.

Hay individuos que insisten en mantener una perspectiva tradicional, en usar como modelos sólo los textos consagrados de los autores más prestigiosos del canon literario. Luego hay los que se enfrentan a esta posición, insistiendo en eliminar ese canon (los famosos «hombres muertos y blancos») y sustituirlo por los «productos culturales» más modernos como el cine, los cómics, los tratados de antropología y medicina, los periódicos y otras muestras de lo que es en realidad, para ellos, la auténtica cultura contemporánea. $\mathrm{Y}$ en el medio hay los que piensan que una 
combinación de estas dos posturas - el mantener la base de textos consagrados y al mismo tiempo abrir la materia a influencias más contemporáneas- representa el auténtico futuro del Hispanismo. Si se añaden a esta mezcla las tensiones creadas sobre el contenido de lo que se va a enseñar, es decir, si esa cultura va a ser la peninsular, la latinoamericana o la US-latina, se puede apreciar la importancia del momento histórico en el que nos encontramos hoy en día.

Estas posibles tensiones tienen dos posibles resultados. O los departamentos de español se adaptan a las nuevas ideas y se abren a las nuevas influencias o se cierran al futuro y se pierden en guerras en las que cada profesor defiende su terreno antiguo (muchas veces bajo el pretexto de «defender los estándares y la excelencia intelectual»). El conflicto es aún mayor al entender que no sólo el qué tiene importancia (es decir, literatura peninsular, latinoamericana, o US-latina) sino también el cómo (acercamientos teóricos como los Estudios Culturales, Estudios de Género -de mujeres, de gays-, Estudios Transatlánticos, Estudios Interdisciplinares, Estudios Comparativos). Todo esto produce tensiones, sí, pero también produce una increíble riqueza de posibilidades y un sentido de futuro en nuestra profesión.

Tradicionalmente, se estudiaban exclusivamente las obras consagradas de los autores consagrados, obras, huelga decir, que merecen su lugar en el canon literario español. Los estudiantes leyeron a los «clásicos» (peninsulares, naturalmente): Juan Ruiz, Jorge Manrique, Celestina, Fray Luis, Quevedo, Cervantes, Calderón, Lope, Zorrilla, Galdós, Unamuno, Azorín, Baroja, Guillén, Lorca, Cela y poco más. Se incluía poco escrito por mujeres (se le indultó a Santa Teresa, quizás a Fernán Caballero y a veces a Laforet), poco de literatura oral (con la excepción de los romances y algún que otro pliego de cordel), nada de periodismo o cultura popular, poco del siglo XVIII, nada de homosexuales (menos Lorca o Goytisolo, pero normalmente no se comentaba su orientación sexual, por no ser «apropiado» o «relevante») y poco de la época moderna (la definición de la cual terminaba con Cela o Delibes). Un autor llegaba a situarse en el «canon» -no se llamaba así hace veinte años; se hacía referencia a «la historia literaria» o a «las listas de lecturas»— con dificultad, y sólo después de muchos años (ver Brown).

La generación de profesores de lengua y literatura españolas que llegó a ocupar las cátedras universitarias en las agitadas décadas de los 60 y 70 se ha jubilado ya o estará jubilada antes de comenzar el nuevo milenio. Los puestos de aquellos grandes profesores -Gonzalo Sobejano, Javier Herrero, Carlos Blanco Aguinaga, Elias Rivers, John H. R. Polt, Claudio Guillén, Russell P. Sebold, Francisco Márquez Villanueva, Bru- 


\section{El Hispanismo que viene: Estados Unidos y Canadá}

ce Wardropper, Monroe Z. Hafter, Geoffrey Ribbans, Rodolfo Cardona, Mario Valdés, Inman Fox, Ciriaco Morón Arroyo y otros más- están ahora en juego, y el desenlace del juego marcará profundamente el hispanismo en los Estados Unidos y Canadá. Los cambios en nuestra profesión en las últimas tres décadas son notables, como comenta Michael McGaha al documentar los temas de las tesis doctorales en el período que cubre 1969-1989: en 1969, un 58 por ciento de las disertaciones leídas en universidades norteamericanas versaban sobre literatura peninsular; en 1989 , solo un 48 por ciento tocaban temas peninsulares (226). Y los cambios serán aún más notables en las próximas dos décadas.

El mismo cambio se nota en los temas que se proponen en las sesiones del congreso anual de la Modern Language Association of America, centro de la actividad universitaria norteamericana en cuanto a lenguas y literaturas extranjeras (e inglesa, claro está). En los últimos cinco años, de 1994 a 1999, se detecta una ligera subida en el número de ponencias dictadas sobre temas latinoamericanos (de 49 en 1994 a 56 en 1999), y una bajada en el número de ponencias leídas sobre temas peninsulares (de 103 en 1994 a 71 en 1999$)^{3}$. El cambio no es tan dramático como en las cifras notadas por McGaha en los temas de las disertaciones, pero esto puede reflejar el hecho de que en general son los profesores más consagrados los que suelen (no exclusivamente, ni mucho menos) participar en las sesiones de la MLA. Es decir, es lógico esperar que dentro de cinco o diez años se darán aún más ponencias sobre temas latinoamericanos (o, como veremos, sobre temas «transatlánticos») y menos sobre temas exclusivamente peninsulares. Ya tenemos indicios de este cambio: en 1994 había una sola ponencia que mezclaba lo peninsular con lo latinoamericano; en 1999 había 12.

Estas estadísticas revelan el importante aumento de «clientes» universitarios en los cursos de español (así se consideran algunos estudiantes y padres de estudiantes que pagan matrículas sustanciosas y por eso exigen más obligación de dar cuentas a la hora de determinar qué se enseña y qué ha aprendido un estudiante en sus años universitarios). Sin embargo, el futuro de la profesión, ahora consolidada en cuanto al número de esos «clientes», no depende ya de tales cifras sino del contenido de lo que se les va a enseñar. Y por eso volvemos a nuestra observación de que estamos en un momento de gran oportunidad si podemos meditar profundamente sobre el futuro de ese "qué» y sacar conclusiones que nos puedan dar la libertad de seguir un curso abierto, flexible y que responda a las necesidades estudiantiles.

Otro indicio de la vitalidad de los estudios hispánicos en los Estados Unidos y Canadá es el impresionante número de publicaciones que salen 


\section{David T. Gies}

de los departamentos de literatura y lingüística españolas. Dar una lista completa de las revistas o de las editoras universitarias o privadas que sacan libros de interés hispanístico sería abusar de la paciencia del lector. Algunas revistas se dedican exclusivamente a estudios peninsulares o a estudios latinoamericanos; otras abren sus páginas a los dos campos o a los estudios US-latinos (aunque sorprende que no existan más). Una breve lista (incompleta) — con el lugar de su publicación y su director/a- incluirá: Afro-Hispanic Review (Missouri, Mullen), Anales Galdosianos (Queen's, Bly), Anales de Literatura Española Contemporánea (Colorado, González del Valle), Arizona Journal of Hispanic Cultural Studies (Arizona, Compitello), Bulletin of the Comediantes (Indiana, Friedman), Caliope (Houston, Olivares), Canadian Journal of Linguistics (Montreal, Rochette), Canadian Modern Language Review (Toronto, Bell), Celestinesca (Michigan State, Snow), Cervantes (Regent's, Eisenberg), Chasqui (Arizona State, Foster), La Corónica (William and Mary, Greenia), Crítica Hispánica (Duquesne, Martín), Cuban Studies (Pittsburgh, Bachman), Dieciocho (Virginia, Gies), Diacritics (Cornell, Resina), España Contemporánea (Ohio State, Amell), Estreno (Ohio Wesleyan, Harper), Gestos (Irvine, Villegas), Hispámerica (Maryland, Sosnowski), Hispania (Georgetown, Irizarry), Hispanic Review (Penn, López), Hispanófila (North Carolina, Clark), Indiana Journal of Hispanic Literatures (Indiana, Sadlier), Journal of Afro-Latin American Studies (Howard, Vieira), Latin American Literary Review (Pittsburgh, Miller), Latin American Theatre Review (Kansas, Woodyard), Letras Femininas (Nebraska, López de Martínez), Letras Peninsulares (Davidson, Vázquez), Modern Language Notes (Johns Hopkins, Sieber), Monographic Review (Texas Tech, Pérez), Nuevo Texto Crítico (Stanford, Ruffinelli), Ojáncano (North Carolina, Gil Casado/Dolgin), Review (Spanish Institute, New York, MacAdam), Revista Canadiense de Estudios Hispánicos (Alberta, Young), Revista de Estudios Hispánicos (Washington, Pope y Sklodowska), Revista Hispánica Moderna (Columbia, Sobejano), Revista Iberoamericana (Pittsburgh, Morana), Romance Notes (North Carolina, Montgomery), Romance Philology (Berkeley, Craddock), Romance Quarterly (Kentucky, Dendle), Romanic Review (Columbia, Riffaterre), Symposium (Syracuse, Bebo) y La Torre (Puerto Rico, Ferinandy).

Joan Ramon Resina observa que «Spanish studies has long been an unappreciated speciality in the anglo-Saxon world» (85) y que lo peninsular ha perdido terreno comparado con las especializaciones latinoamericanas. Sugiere este crítico una manera de volver a recuperar el equilibrio en los estudios hispánicos: 


\section{El Hispanismo que viene: Estados Unidos y Canadá}

If the Latin American side boosts the enrollment and provides the density necessary for a modicum of administrative respect, the Peninsular side provides a historical weight and a European connection, which might yet prove invaluable in challenging the supercilious hegemony of English departments and winning for Hispanism the status of world culture that it has always coveted. (118)

El estudio de Resina explica con gran perspicacia las raíces conservadoras del hispanismo norteamericano (basado en la filología germánica decimonónica), que transforma la identidad «española» en «castellana»; Resina intenta reivindicar el lugar que debe tener (pero que no tiene, por razones que explica) la literatura catalana en el canon hispánico. Concluye Resina que el hispanismo norteamericano es «a house divided against itself» (115) porque los practicantes de la disciplina se demuestran demasiado propensos a «jump onto any programmatic or terminological wagon that seems to run» (115). Este pluralismo crea «disarray» (115) en su opinión; hasta cierto punto, como veremos, tiene razón.

Una encuesta informal y poco científica - pero reveladora- sobre el paso gradual de los estudios peninsulares a los estudios latinoamericanos en las mejores universidades estadounidenses y canadienses indica que lo que hemos visto arriba en cuanto a las disertaciones y a las intervenciones en el congreso anual de la MLA refleja con cierta precisión la realidad de hoy. Resina cree que el estudio de la cultura peninsular cayó en la «irrelevancia» durante la Guerra Fría de los años 1950, y que los estudios latinoamericanos llegaron para llenar el vacío (117); este cambio, según su modo de ver, creó tensiones y competición interna que «has not helped the discipline, even if it has brougth "moral" and material triumphs to its Latin American fraction» (117). Interpretado desde otra perspectiva, este cambio sugiere o 1) que los «antiguos» campos peninsulares pierden terreno frente a los «nuevos» campos latinoamericanos y US-latinos o 2) que los estudios latinoamericanos por fin alcanzan paridad con los estudios peninsulares, es decir, que empieza a manifestarse un equilibrio en los departamentos de español entre lo peninsular y lo latinoamericano. Creo que el observador más objetivo verá que es lo segundo, es decir, que el aumento del interés por los estudios latinoamericanos ha dado la oportunidad a muchos departamentos de reforzar y aumentar el número de profesores que enseñan literatura latinoamericana y así ofrecer más cursos sobre esas materias.

Algunas veces este aumento del número de profesores que enseñan estudios latinoamericanos se produce a expensas de la sección peninsular, pero no siempre. Por dar un solo ejemplo, en la Universidad de Virginia se ha visto la adición de una nueva línea docente (es decir, un nue- 
vo puesto) dedicado a la enseñanza de literatura latinoamericana, pero sin la disminución del número de profesores que enseñan literatura peninsular. Un aumento del número de profesores con permanencia no se consigue fácilmente y no es la solución más frecuente hoy en día, cuando las universidades suelen reducir el número de profesores permanentes. En algunas universidades (Virginia, Ohio State, Yale) la jubilación de un profesor dedicado a lo «peninsular» abre paso a otro profesor peninsular, pero en otras muchas universidades (Berkeley, Vanderbilt, SUNY Stony Brook, Indiana, Emory, New York University, Minnesota, Denver, Indiana, Alberta), el puesto peninsular se ha trasladado al campo latinoamericano, dando lugar a un reajuste entre la sección peninsular y la sección latinoamericana. A veces, al trasladar un puesto peninsular a lo latinoamericano, se pierde completamente un campo de estudios peninsular (medieval, Siglo de Oro, etc.) porque no queda nadie para dirigir tesis doctorales en dicho campo, algo que amenaza el balance tradicional. Varias universidades han recurrido a otra solución, que es reemplazar a un profesor jubilado por dos profesores jóvenes, dividiendo así una línea en dos, una para estudios peninsulares y otra para estudios latinoamericanos o US-latinos. Otra «solución» es buscar a un joven profesor que pueda dictar clases sobre los dos campos, es decir, que pueda combinar, por ejemplo, la poesía peninsular con la latinoamericana o el período colonial tanto en la península como en Latinoamérica. Otra opción más, al jubilarse un profesor peninsular, consiste en la simple reducción del plantel profesoral (Universidad de Connecticut, por ejemplo). Resina ve la oportunidad presentada:

As far as the Spanish government is concerned, the Hispanic population of the U.S. is important, not for the preservation of a marginal community of Peninsular scholars, but as a potential market of significant proportions for Spanish products and services, provided the link to Spain is there. In terms of legitimation, however, the relation between the Latin American and the Spanish sides of the discipline works reciprocally, as the more perspicacious among Hispanists have always known. (118)

La rígida división tradicional - literatura peninsular versus literatura latinoamericana - ya está en vías de desaparecer, como hemos notado arriba, no sólo por la llegada e influencia de los partidarios de los estudios US-latinos, sino también por el reconocimiento de que las antiguas categorías rígidas ya no sirven y no nos servirán en el futuro. Ya no es válido (o útil) hablar del hispanismo peninsular vs. americano. Ahora, los mejores departamentos buscan cómo combinar las dos categorías, o cómo enriquecerlas con otras perspectivas críticas u otras lite- 


\section{El Hispanismo que viene: Estados Unidos y Canadá}

raturas. Una nueva disciplina que se ha llamado «Estudios Transatlánticos» comienza a atraer atención y estudiantes (Harvard, Stanford, Virginia). Resina, al pedir no menos «hispanismo» sino más «hispanismos» (aunque para insistir en que se incluya el estudio de la cultura catalana, 121), reconoce que el futuro va a ser un lugar multi-disciplinar y abierto a muchas novedades.

Lo mismo pasa en Canadá, donde los grandes centros de estudios hispánicos - Montreal, McGill, Alberta, British Columbia y la más prestigiosa, Toronto - han visto un cambio en los intereses intelectuales del profesorado y una mayor demanda de especialistas en latinoamericana (Alberta, por ejemplo, se concentra en estudios Latinoamericanos). Hay que notar, sin embargo, que aunque son pocos los departamentos que dan el doctorado, los programas de Master's en las universidades canadienses (Queen's, Western, Ottawa) suelen ser más rigurosos y más especializados que los que se dan en las universidades estadounidenses.

Todo esto refleja el contenido de la disciplina, el qué se va a estudiar, el contenido de los cursos y de las lecturas obligatorias, que evoluciona continuamente. Otra evolución - acaso revolución (lo que George Mariscal llama «la revolución teórica de los últimos veinticinco años» 1)- tiene lugar en el cómo se estudia ese contenido, es decir, desde qué perspectiva o perspectivas. El panorama de perspectivas críticas es riquísimo hoy en día en los Estados Unidos y Canadá. Por dar sólo una lista muy corta, en las universidades norteamericanas uno puede encontrar practicantes de movimientos críticos como los «Estudios Culturales,» «Estudios Gay y Lesbianos,» «Teoría de la Representación» (Performance Theory), «Feminismo,» «Nueva Crítica Americana,» «Nuevo Historicismo,» «Deconstrucción,» «Postestructuralismo,» «Hermenéutica,» «Estudios Poscoloniales,» «Marxismo» y ese cajón de sastre teórico, «Posmodernismo.» El panorama es amplio, vigoroso y , no hay que decirlo, conflictivo.

En 1967 sólo un 13 por ciento de los doctorados concedidos en universidades estadounidenses y canadienses fueron a mujeres; en 1999 ha aumentado hasta un 41 por ciento. Este espectacular ascenso del número de mujeres que participan en el campo de los estudios hispánicos trae consigo un cambio en la manera de conceptualizar la profesión. La revolución feminista de los años 70 produjo una revolución crítica también en las universidades, una nueva manera de leer la literatura, de analizar el discurso literario, de abrir el canon que estudiamos y de teorizar sobre la contribución de la mujer a la cultura occidental. Si todo esto comenzó en los campos de la literatura y la lingüística inglesas y francesas, pasó en breve al hispanismo y el panorama de acercamientos críticos que ahora se llaman «feministas» ha enriquecido profundamente la profesión. La 
crítica feminista es mucho más de lo que sus detractores creen; no es, ni mucho menos, una perspectiva limitada ni fanática (así lo afirman los que se oponen a los partidarios de dicho acercamiento), sino un rico panorama de acercamientos críticos que intenta descubrir en determinados textos lo que aporta el género femenino, como sujeto o como objeto, a su interpretación. En los Estados Unidos la crítica literaria feminista va unida al movimiento feminista, fenómeno que alcanzó su punto más llamativo durante la década de los 1970. «El objeto concreto del estudio puede ser el tratamiento de lo femenino en los textos escritos por hombres o mujeres, la relación entre la vida de la escritora, su contexto social y su obra, o la diferencia femenina desvelada por las escritoras y sus lectoras y críticas» (Scarlett 91). Acierta Loureiro al escribir que «el feminismo, a pesar de todas las dificultades teóricas con las que se enfrenta, [es] una de las grandes aportaciones de la teoría contemporánea» (34). El feminismo ha estimulado un sinnúmero de publicaciones, congresos y cursos sobre mujeres (o que incluyen mujeres) que han revitalizado todos los campos hispánicos. Si ahora esa crítica feminista va disminuyendo como enfoque crítico, lo que hemos aprendido de ese acercamiento se transforma en una visión, quizás algo más amplia, llamada «Gender Studies,» un campo que se está abriendo tanto a las mujeres como a los hombres. Los «Gender Studies» se combinan, por ejemplo, con los llamados «Queer Studies» o «Gay Studies,» un campo ocupado por estudiosos de ambos sexos.

Sin embargo, el aumento del número de mujeres como estudiantes y como profesoras no ha creado un equilibrio en los rangos más prestigiosos de los departamentos de español; es decir, aunque más del 50 por ciento de los alumnos en los cursos de doctorado son mujeres, sólo un 20 o 25 por ciento de mujeres han accedido a los puestos con permanencia o a las cátedras más prestigiosas. Este lamentable desequilibrio poco a poco va cambiando, pero los cambios son lentos, más lentos de lo que muchos desean.

Otro movimiento de gran interés en los Estados Unidos y Canadá hoy en día son los «Cultural Studies» (Estudios Culturales), que toman como su punto de partida una postura que intenta iluminar la literatura desde una perspectiva más amplia que la que dominaba los campos literarios estadounidenses durante muchos años (»Nueva Crítica Americana» o la tradicional filología hispanística). «Los Estudios Culturales —opina Del Pinose alejan del formalismo y de la preeminencia del texto literario como el objeto primordial de análisis crítico. Sin dejar de prestar atención a la literatura - que en general resulta más privilegiada que otras manifestaciones artísticas-, el foco de atención se desplaza hacia la variedad de productos culturales elaborados por una sociedad bajo unas condiciones concretas. 
Consecuentemente, ello conduce a un predominio del componente interdisciplinar» (255). Es decir, la literatura es una «mera» manifestación cultural entre otras muchas que merecen nuestra atención. Aunque parece ser «nuevo» este movimiento, en realidad es un redescubrimiento de una teoría propuesta en Inglaterra en los años sesenta, elaborada y enriquecida por el feminismo y por los estudios poscoloniales. Comenzó a influir en las universidades norteamericanas a finales de los años ochenta y los principios de los años noventa, aunque para Jo Labanyi, los Estudios Culturales todavía están en su primera juventud (v). Sigue en boga e incluso va creciendo el interés por la producción cultural (cine, medios de comunicación) más que por el objeto estrictamente literario.

Los Estudios Culturales han extendido el ya agotado postestructuralismo y lo han enriquecido con el feminismo, el poscolonialismo y el nuevo historicismo para producir un fenómeno difícil de definir. Los mejores cultivadores de los Estudios Culturales toman en cuenta el texto (varios textos, incluyendo los periódicos, el cine y los cómics) y su contexto (historia, conflicto de clases, nacionalismo, género, sexualidad, ideología, raza, literatura "alta" vs. literatura de masas) al analizar una obra determinada. Es decir, como ha subrayado Del Pino, es un campo interdisciplinar. Esto no significa, ni mucho menos, que tales cosas no se tomaran en cuenta antes al analizar un texto (los críticos marxistas siempre han leído la literatura desde la perspectiva del conflicto de clases y la ideología dominante, y los llamados críticos «tradicionalistas» siempre leyeron los textos dentro de su contexto histórico), pero ahora en vez de intentar evitar la contradicción producida por la lectura múltiple de un texto $\mathrm{y}$ el intento de someterla a una teoría unificadora, los críticos culturales abrazan y a veces incluso buscan contradicciones textuales. En las palabras de Stephanie Sieburth, «Until relatively recently, modern peninsular Hispanism, like all literary studies influenced by the New Criticism, used the narrow frame of "the general body of the arts" as a field of study. Of course, critics related literature to the political events of the day, but in relatively broad strokes [...] In the last twenty years, the few focus on mass cultural forms such as serial novels, radio songs, television programs, movies, and the like has led scholars to ask radically new questions of the literary works they had been studying all along, as well as leading them to new objects of study» (13-14). Así, un texto es el resultado de un proceso cultural.

Lo mejor de los Estudios Culturales es que los investigadores han descubierto el valor de combinar la teoría con el trabajo textual y trabajo de archivos y han tenido que volver a los documentos de la época para iluminar el texto comentado. Si en los años sesenta y setenta muchos crí- 
ticos universitarios defendían o "el texto y nada sino el texto» o la pura teoría (muchas veces sin mencionar ni un solo texto), durante los años ochenta y noventa combinan lo mejor de aquellas posturas. Como escribe Joseba Gabilondo, los Estudios Culturales han atraído la atención de los profesores en los Estados Unidos por razones históricas: «Así, la atención dedicada por los Estudios Culturales a problemas raciales, postcoloniales y de exilio han facilitado su incorporación al contexto norteamericano donde dichos temas se perciben como de vital importancia teórica» (232). Aunque el interés por los Estudios Culturales aumentará en la próxima década en el campo peninsular, es en el campo de la literatura latinoamericana donde han tenido más influencia, especialmente si los vemos como complemento o extensión del posmodernismo o poscolonialismo. Pero, como ha comentado un colega de otra universidad, «Todo el mundo ahora intenta "hacer" los Estudios Culturales pero nadie parece entender exactamente qué son ni cómo se hacen", confusión que puede aclararse con la inminente publicación de una nueva revista, Journal of Spanish Cultural Studies, que saldrá pronto en Inglaterra (Royal Holloway) y los Estados Unidos (Duke).

El Posmodernismo ha dominado el discurso crítico en los Estados Unidos desde el declive del posestructuralismo en los años 80. Del posestructuralismo los críticos aprendieron que los textos no viven aislados de una red de significados múltiples; los posmodernistas intentan explicar cómo aquellos significados se sitúan dentro de un marco «transtemporal y multidireccional» (Navajas 149). No es nada fácil definir el posmodernismo, y pocos críticos han sido tan atrevidos como para elaborar una definición sintética. Sin embargo el acercamiento posmodernista suele incluir un análisis de obras que privilegian lo lúdico sobre lo intencional, la metonimia sobre la metáfora, la ausencia sobre la presencia, la anti-narrativa sobre la narrativa (en el sentido de las pequeñas historias contra las grandes historias), la indeterminación sobre la determinación, la ironía sobre la metafísica, el simulacro sobre la realidad concreta, el pastiche sobre la narración cronológica, y lo marginal sobre lo central (ver Hassan).

Estudiosos de los Estudios Gay y Lesbianos, abiertos quizás a partir del interés por el Feminismo y los Estudios Culturales, han creado miniescuelas en varias universidades que abren paso a un nuevo acercamiento a la literatura hispánica, lo que James Mandrell llama «la. creación de un espacio adecuado para una lectura específica y abiertamente orientada hacia una perspectiva gay y lésbica [y] la interpretación de textos específicamente gay y/o lesbianos» (211). Para Mandrell, la rúbrica «estudios gay y lesbianos indica una conciencia, una identidad política, 
social y cultural moderna, incluso una cultura particular» (214) que los conecta desde cierta perspectiva, con los Estudios Culturales o los Estudios de Género. Recientemente, editoriales universitarias de gran prestigio como Duke University Press han sacado libros con títulos llamativos como ¿Entiendes? Queer Reading, Hispanic Writings o Queer Iberia, testimonio de las nuevas tendencias críticas que empiezan a estar de moda en los Estados Unidos.

A pesar de este ejemplo, la publicación de libros de tema hispánico es mucho más difícil hoy en día que en los años del florecimiento de los estudios hispánicos en los Estados Unidos y Canadá. Es decir, la mayoría de las editoras universitarias norteamericanas ahora publican pocos estudios de seria investigación literaria o cultural por la sencilla razón de que se venden poco. Es un círculo vicioso: los libros se venden poco porque son caros o porque las bibliotecas universitarias han sufrido importantes recortes en sus presupuestos; compran menos libros y por eso se publican menos libros. Hay excepciones. Varias editoriales universitarias sacan una respetable cantidad de libros hispánicos (de tema tanto peninsular como latinoamericano) - Penn State, Arizona, Missouri, Purdue, Bucknell, Illinois, Texas, Vanderbilt-o publican de vez en cuando un libro sobre un tema literario, histórico o cultural hispánico - Albany, Nebraska, Pennsylvania, Puerto Rico, North Carolina, Toronto o Yale. Algunas editoriales independientes, pero muchas veces relacionadas con individuos que enseñan en departamentos de español, como el Bilingual Review Press, Curbstone Press, Dalky Archive Press, Dovehouse Editions, Ediciones del Norte, Ediciones Universal, Juan de la Cuesta o Scripta Humanistica, también se concentran en obras de interés hispánico. Un fenómeno notable - el Arte Público Press, de Houston- se ha convertido en la editorial más importante de literatura US-latina. Arte Público, fundado por Nicolás Kanellos en 1979, publica treinta títulos al año, la gran mayoría en inglés, para «recuperar la tradición hispánica» y "proporcionar una voz a los escritores de descendencia y herencia hispana» en Estados Unidos.

Los mejores departamentos de estudios hispánicos no pueden, ni quieren, hacerlo todo. Algunos intentan mantener un equilibrio entre lo peninsular y lo latinoamericano, mientras que otros abogan por una perspectiva más concentrada en uno u otro, o en un acercamiento teórico que predomine sobre los demás (Duke, por ejemplo, se concentra ahora en los Estudios Culturales). Algunos departamentos también han mantenido una línea rigurosa de estudios lingüísticos (minoritaria, ciertamente), es decir, la enseñanza de la filología/linguística histórica, la lingüística generativa, la lingüística aplicada/pedagógica y la socio- 
lingüística, que siempre han sido elementos fundamentales en lo que es el «Hispanismo.» Esta filología tiene sus partidarios más activos en Craddock (Berkeley), Dworkin (Michigan), Rini (Virginia), Walsh (Georgetown), Pharies (Florida), Wanner (Ohio State), Lipski (New Mexico), Harris-Northall (Wisconsin) y Lloyd (Pennsylvania). La sociolingüística se enseña en la Universidad de California del Sur (USC, Silva-Corvalán), entre otros sitios, mientras que la lingüística generativa (escuela iniciada por Chomsky) tiene sus partidarios en Nuñez-Cedeño (Illinois, Chicago), Zamora (Massachusetts), Contreras (Washington), Zagona (Washington) y Harris (MIT).

En 1995 el National Research Council publicó los resultados de una encuesta científica y de gran impacto que compuso un ranking de los «mejores» departamentos de estudios hispánicos en los Estados Unidos. La lista de los departamentos de español en las universidades públicas fue: Virginia, Wisconsin, Berkeley, Kansas, Texas y Michigan. La lista definitiva, que incluía todas las universidades, tanto públicas como privadas, rezaba así: Columbia, Duke, Brown, Princeton, Virginia, Pennsylvania, Wisconsin, Cornell, Berkeley, Harvard, Kansas, Texas y Michigan. Pero aquella lista podría fácilmente incluir otros centros importantes, donde el hispanismo es activo, fuerte y de gran vigor intelectual como New York University, Emory, Indiana, Arizona, Arizona State, North Carolina, Yale, Vanderbilt, Ohio State, o Illinois.

Uno de los grandes aciertos del sistema universitario norteamericano es su flexibilidad. Es decir, los cambios de acercamiento teórico, de listas de lecturas obligatorias, o de métodos pedagógicos pueden efectuarse con rápidez. Esta flexibilidad (u oportunismo, como dirían algunos) ofrece la posibilidad de rápidos cambios en el contenido y dirección de los departamentos de español. No todo es «desolación y miseria» en el hispanismo, como el interesantísimo "panfleto autobiográfico» (32) de Ángel Loureiro propone al reseñar el libro Critical Practices in Post-Franco Spain, donde critica (con razón) «la estructura rígida, autárquica, endogámica y jerarquizada que todavía domina en la universidad española» (33). Al contrario.

Si en España las cosas van demasiado despacio - y en los departamentos de filología parecen no moverse-, en los Estados Unidos las cosas parecen ir demasiado deprisa en lo que respecta a la sucesión y el descarte de acercamientos teóricos. Frente a la endogamia incestuosa del hispanismo español nos las vemos aquí con la búsqueda de una constantemente nueva, última frontera americana [...] Y para complicar las cosas todavía más, el hispanismo norteamericano sigue, como siempre, a la cola de la vanguardia. mimetizando a trancas y barrancas lo que parece estar de moda, 
El Hispanismo que viene: Estados Unidos y Canadá

obligado a ir siempre demasiado deprisa y llegando siempre demasiado tarde. (34)

Pero para Luis Beltrán, el resultado es el dominio de una investigación literaria norteamericana en la que se aprecia «un individualismo radical» $\mathrm{y}$ una tendencia a la diversidad propulsada "por una dinámica social centrífuga, basada en el plurilingüismo y en la diversidad cultural. El resultado es un panorama de la investigación literaria más plural» (46).

Esta pluralidad, como ya hemos dicho, enriquece el panorama del hispanismo en los Estados Unidos y Canadá. Sin embargo, los hispanistas no somos futurólogos y por eso es difícil, si no imposible, pronosticar hacia dónde irá el hispanismo en el futuro y cuáles serán las tendencias más importantes. No obstante, podemos descifrar varias tendencias actuales que pueden influir en la dirección de nuestra profesión. A pesar de la negatividad expresada por algunos colegas (especialmente, los que han sufrido los rápidos cambios en la profesión en los últimos veinte años y que definen lo que es el hispanismo norteamericano desde una perspectiva ya anticuada), el futuro ofrece muchas posibilidades. Esto no quiere decir que los problemas sean pequeños y fácilmente superables. Es cierto que los estudios graduados ya no existen en la misma forma en que los conocimos los que ya estamos en la cumbre de la profesión y que ocupamos las cátedras más importantes. La gradual reorganización de las universidades; la división entre las universidades privadas y las públicas; la tendencia a contratar a profesores a tiempo parcial; el deseo de reducir gastos; la redistribución de áreas de concentración entre lo peninsular, lo latinoamericano y lo US-latino; la dificultad de publicar monografías o libros de investigación en las grandes editoras universitarias - todo esto puede amenazar el vigor de la profesión y desanimar a los jóvenes que buscan hacer carrera en la enseñanza universitaria-. Pero, como pasa muchas veces, al cerrarse una puerta se abre otra. El número de estudiantes que quiere estudiar el español (tanto la lengua como la literatura y la cultura) crece espectacularmente y eso produce mejores estudiantes en los niveles más avanzados. Siguen solicitando puestos en los mejores programas graduados estudiantes que han demostrado mucho talento y mucho interés por el hispanismo. Siguen colocándose en importantes departamentos de español. La revolución tecnológica ha producido una generación de estudiantes que quiere aplicar dicha tecnología al estudio de los textos tradicionales. El fervor crítico de las tendencias que dominan el discurso crítico de los años 90 - posmodernismo, poscolo- 
nialismo, estudios de género, estudios gays, estudios culturales, estudios interdisciplinares - nos abre nuevos panoramas y nuevas posibilidades para nuestra investigación de la lengua, literatura y cultura hispánicas.

El futuro del hispanismo norteamericano - el reto, si se quiere- depende de muchos factores. Si nuestra profesión se adhiere a las antiguas divisiones y categorías que han dominado el discurso literario durante los últimos cuarenta años, ese futuro consistirá en que los profesores de literatura tendrán que someterse a las realidades del mercado, convirtiéndose en instructores de lengua, luchando contra sus colegas peninsulares o latinoamericanos o US-latinos para atraer a los estudiantes a sus clases, sentenciados a la enseñanza básica (cursos de lengua para los estudiantes de negocios, español como disciplina puramente secundaria y "práctica» para los que quieren conquistar más mercados tanto en los Estados Unidos como en Latinoamerica), o defendiendo su propio centrismo europeo/peninsular, latinoamericano o US-latino. Es decir, llegaremos plenamente a esa «irrelevancia» de que habla Resina. O peor, a la extinción.

Hoy estamos en el momento idóneo para reflexionar sobre la profesión y crear un futuro lleno de oportunidades. Una pregunta fundamental la plantea Idelber Avelar:

How do we rethink the role of Spanish programs in the era of the dissemination of critical theory, the Latinization of the United States, the culturalization of literature, the globalization of culture, and the permeabilization of national and disciplinary borders? (50)

Lo que implica esta pregunta es la demolición de las antiguas categorías geográficas que han domin do los departamentos de español durante décadas. La posibilidad de eliminar (o por lo menos disminuir) la geografía o la cronología como los únicos determinantes nos abrirá a la posibilidad de más flexibilidad interdisciplinar, por ejemplo, a la ampliación de otras categorías como "Early Modern Spain», "La Vanguardia», «Estudios Transatlánticos», «llustración y Colonialismo», "Hispanismo/ Spanish en las Américas», «Modernidad» y otras.

Este es nuestro reto: re-pensar la esencia del hispanismo norteamericano, las estructuras departamentales y universitarias, descubrir nuevas conexiones interdisciplinares no sólo entre los varios campos de hispanismo (peninsular, latinoamericano, US-latino) sino entre el hispanismo y el «más allá» antropológico, histórico, psicológico o teórico. Si el hispanismo ha aceptado un lugar en el furgón de cola teórico (ver el artículo de Alonso 


\section{El Hispanismo que viene: Estados Unidos y Canadá}

sobre el lugar «incómodo» que ocupa la «teoría» en los estudios hispánicos norteamericanos), es hora de inventar una teoría del hispanismo en vez de «aplicar» las teorías heredadas de fuera (Francia, sobre todo). Si el hispanismo ha sido un campo marginado (¿auto-marginado?) en las universidades norteamericanas -en el sentido de no participar como presencia activa o iniciadora en los programas de literatura comparada o interdisciplinares ${ }^{4}$ - es hora de romper aquel provincianismo y crear escuelas teóricas que salgan de nuestros textos y departamentos en vez de venir a colonizarlos. Si el hispanismo ha resistido los nuevos desafíos que implica el reajuste de balance entre los varios segmentos intelectuales de nuestra disciplina, es hora de ver el futuro con entusiasmo y no con miedo.

Si sabemos entablar nuevos diálogos con nuestros colegas (tanto en los mismos departamentos como en otras facultades), repensar lo que es el Hispanismo en los Estados Unidos y Canadá, y demostrar más creatividad a la hora de conceptualizar la profesión, las oportunidades serán infinitas y el futuro estará asegurado.

\section{Notas}

* Quisiera agradecer a los muchos colegas en los Estados Unidos y Canadá que han contestado preguntas, ayudado con las encuestas y ofrecido valiosas correcciones sobre esta materia durante la larga preparación de este artículo. A todos mis más sinceros agradecimientos.

1 De aquí en adelante, se empleará la palabra "US-latina» o "US-latino" para referirse a los "Latino Studies" en los Estados Unidos. Naturalmente, no hay que decir que no tiene nada que ver con la disciplina de literatura clásica (latina o griega). Versan sobre el estudio de la literatura escrita por la minoría hispana en los Estados Unidos, tanto en inglés como en español.

2 Aquí usamos la palabra "filología" para incluir todos los campos de literatura y lingüística española, no para describir el acercamiento tradicional a los estudios literarios. En la opinión de algunos practicantes, las nuevas metodologías suplementan, no complementan, la filología tradicional en los departamentos de español. El único departamento que hoy en día todavía concede un certificado específicamente titulado "filología" es Berkeley.

3 El descenso del número de ponencias sobre literatura peninsular no es tan marcado como sugiere esta trayectoria. Las cifras anuales son: 1994 (49 ponencias latinoamericanas, 103 peninsulares, 1 mixta), 1995 (59 latinoamericanas, 70 peninsulares, 2 mixtas), 1996 (62 latinoamericanas, 102 peninsulares, 5 mixtas), 1997 (45 latinoamericanas, 105 peninsulares, 4 mixtas), 1998 (74 latinoamericanas, 98 peninsulares, 6 mixtas), 1999 (56 latinoamericanas, 71 peninsulares, 12 mixtas).

4 Avelar afirma que «Established by the Iberian stylistic and philological schools United States Hispanism until very recently was, and to a great extent still is, virulently antispeculative and antitheoretical» 51. 


\section{Obras citadas}

Alonso, Carlos. "Cultural Studies and Hispanism: Been There, Done That." Siglo XX/Twentieth Century 14 (1996): 137-151.

AvelAR, IDELBER. «The Clandestine Ménage à Trois of Cultural Studies, Spanish, and Critical Theory." Profession 1999. Ed. Phyllis Franklin. New York: The Modern Language Asssociation of America, 1999. 49-58.

Beltrán Almería, Luis. "La filología hispánica en la encrucijada.» Quimera 139 (1995): 45-49.

Brown, JOAN L. and Crista Johnson. «The Contemporary Hispanic Novel: Is There a Canon?» Hispania 78 (1995): 252-261.

Silvia L. López, Jenaro Talens, y Darío Villanueva. Critical Practices in Post-Franco Spain. Ed. Minneapolis: U Minnesota P, 1994.

Emilie L. Bergmann y Paul Julian Smith. ¿Entiendes? Queer Readings, Hispanic Writings. Ed. Durham: Duke UP, 1995.

GABILONDO, JOSEBA. «Estudios culturales:"Travestismo y novela terrorista: Masoquismo femenino y deseo en la literatura vasca postnacional"». En El hispanismo en los Estados Unidos. Discursos críticos / prácticas textuales. Ed. José M. del Pino y Francisco La Rubia-Prado. Madrid: Visor: 1999. 231-254.

Hassan, Inab. The Postmodern Turn. Essays in Postmodern Theory and Culture. Columbus, OH: Ohio State UP, 1988.

LABANYI, Jo. «Editor's Preface.» En Spanish Cultural Studies: An Introduction. Ed. Helen Graham y Jo Labanyi. Oxford: Oxford University Press, 1995. v-viii.

LOUREIRO, ÁNGEL. «Desolación y miseria del hispanismo.» Quimera 139 (1995): 31-36.

MANDRELL, JAMES. «Estudios gay y lesbianos: "La revelación del cuerpo masculino: una mirada gay”.» En El hispanismo en los Estados Unidos. Discursos críticos / prácticas textuales. Ed. José M. del Pino y Francisco La Rubia-Prado. Madrid: Visor: 1999. 211-230.

MARISCAL, GEORGE. "An Introduction to the Ideology of Hispanism in the US and Britain.» En Conflicts of Discourse: Spanish Literature in the Golden Age. Ed. Peter W. Evans. Manchester: Manchester UP, 1990. 1-25.

MCGAHA, MiCHAEL. «What Ever Happened to Hispanism?» Journal of Hispanic Philology 14 (1990): 225-230.

Morales, Amparo. «Tendencias de la lengua española en Estados Unidos.» El español en el mundo. Madrid: Instituto Cervantes / Plaza y Janés, 1999. 241-272.

NAvajas, GonZalo. "Posmodernismo: “¿Cómo leer una novela hoy?”.» En El hispanismo en los Estados Unidos. Discursos críticos / prácticas textuales. Ed. José M. del Pino y Francisco La Rubia-Prado. Madrid: Visor: 1999. 149-167.

PINO, JosÉ M. DEL. «Estudios culturales: "Sujeto, nación y estereotipos de marginalidad: Los gitanos en la cultura española del siglo veinte"." En El hispanismo en los Estados Unidos. Discursos críticos / prácticas textuales. Ed. José M. del Pino y Francisco La Rubia-Prado. Madrid: Visor: 1999. 255-276.

Queer Iberia. Sexualities, Cultures, and Crossings from the Middle Ages to the Renaissance. Ed. Josaih Blackmore and Gregory S. Hutchinson. Durham: Duke UP, 1999.

Resina, JoAn Ramon. “Hispanism and Its Discontents.» Siglo XX / 20th Century 14. 1-2 (1996): 85-135.

SCARLETT, ElizABETH. «Feminismo: "La crítica feminista en el hispanismo, la recepción feminista de Rosalía de Castro y la melancolía femenina en En las orillas del Sar'.» En 


\section{El Hispanismo que viene: Estados Unidos y Canadá}

El hispanismo en los Estados Unidos. Discursos críticos/prácticas textuales. Ed. José M. del Pino y Francisco La Rubia-Prado. Madrid: Visor: 1999. 91-109.

Sieburth, Stephanie. «What Does It Mean to Study Modern Spanish Culture?» En The Cambridge Companion to Modern Spanish Culture. Ed. David T. Gies. Cambridge: Cambridge University Press, 1999. 11-20. 